\title{
Lipocalina associada à gelatinase de neutrófilos (NGAL) e calprotectina no tecido laminar de equinos após obstrução jejunal, tratados ou não com hidrocortisona ${ }^{1}$
}

\author{
Luciane M. Laskoski ${ }^{2 *}$, Carlos A.A. Valadão², Rosemere 0. Vasconcelos ${ }^{3}$, Rafael R. Faleiros ${ }^{4}$, \\ Heloisa M.F. Mendes ${ }^{4}$, Danilo Ferrucci ${ }^{5}$, Juliete A.F. Silva ${ }^{5}$ e Dagmar D.R.S. Machado ${ }^{5}$
}

\begin{abstract}
Laskoski L.M.,Valadão C.A.A., Vasconcelos R.O., Faleiros R.R., Mendes H.M.F., Ferrucci D., Silva J.A.F. \& Machado D.D.R.S. 2012. [Neutrophil gelatinase associated lipocalin (NGAL) and calprotectin in equine laminar tissue after jejunal obstruction, treated or not with hidrocortisone.] Lipocalina associada à gelatinase de neutrófilos (NGAL) e calprotectina no tecido laminar de equinos após obstrução jejunal, tratados ou não com hidrocortisona. Pesquisa Veterinária Brasileira 32(9):817-823. Departamento de Clínica e Cirurgia Veterinária, Faculdade de Ciências Agrárias e Veterinárias, Universidade Estadual Paulista, Prof. Paulo Donato Castellane s/n, Jaboticabal, SP 14884-900, Brazil. E-mail: luciane-laskoski@hotmail.com

Laminitis is a severe hoof condition in horses that may cause intense suffering. In this study, leukocyte infiltration in hoof laminar tissue was investigated in horses subject to intestinal obstruction using immunohistochemistry to detect calprotectin, and zymography to detect neutrophil gelatinase associated lipocalin (NGAL). There were four groups: the Control Group (Gc), with seven horses, without surgical procedures; the Sham-operated Group (Gi), with five horses that were subjected to surgical procedure without intestinal obstruction; the No Treat group (Gnt), with four horses subjected to intestinal obstruction (jejunal distention using an intraluminal balloon) without treatment; and Treated group (Gt), with four horses subjected to intestinal obstruction and treated with hydrocortisone. Positive calprotectin imunostaining was detected in all experimental groups, with increase cell counts in horses of the distended group compared with the control group. NGAL expression was increased in Gd compared with Gc e Gi. The Gt did not differ from the others. In conclusion, small intestine distension can promote leukocyte infiltration in equine hoof laminar tissue, and NGAL zymography was considered a useful method for leukocyte tissue detection in horses. New studies will be conducted to verify the possible beneficial anti-inflammatory effects of hydrocortisone in hoof of horses with intestinal obstruction.
\end{abstract}

INDEX-TERMS: Horse, laminitis, calprotectin, NGAL, jejunal obstruction.

RESUMO.- A laminite é uma doença podal grave que acomete os equídeos, sendo responsável por intenso sofrimento. Neste estudo foram pesquisadas a presença de cal-

\footnotetext{
${ }^{1}$ Recebido em 26 de outubro de 2011.

Aceito para publicação em 8 de março de 2012.

${ }^{2}$ Departamento de Clínica e Cirurgia Veterinária, Faculdade de Ciências Agrárias e Veterinárias (FCAV), Universidade Estadual Paulista (Unesp), Prof. Paulo Donato Castellane s/n, Jaboticabal, SP 14884-900, Brasil. *Autor para correspondência: luciane-laskoski@hotmail.com

${ }^{3}$ Departamento de Patologia Veterinária, FCAV-Unesp, Jaboticabal, SP.

${ }^{4}$ Departamento de Clínica e Cirurgia Veterinária, Universidade Federal de Minas Gerais (UFMG), Av. Antônio Carlos 6627, Belo Horizonte, MG 30123-970, Brasil.

${ }^{5}$ Departamento de Imunologia Aplicada, Universidade de Campinas (Unicamp), Rua Monteiro Lobato s/n, Campinas, SP 13083-862, Brasil.
}

protectina por meio da imunoistoquímica, e de lipocalina associada à gelatinase de neutrófilos (NGAL), por zimografia, no tecido laminar do casco de equinos após obstrução intestinal. Os animais foram divididos em quatro grupos: Grupo controle $(\mathrm{Gc})$, contendo sete animais normais, sem procedimento cirúrgico; Grupo Instrumentado (Gi), contendo cinco animais, os quais passaram por todo o procedimento cirúrgico sem sofrerem obstrução intestinal; Grupo Não Tratado (Gnt), contendo quatro equinos submetidos a obstrução intestinal do jejuno por distensão de balão intraluminal, sem tratamento; e Grupo Tratado (Gt), contendo quatro equinos submetidos a obstrução intestinal, e tratados preventivamente com hidrocortisona. Houve imunomarcação de calprotectina em todos os grupos experimentais, com aumento nos equinos do grupo distendido 
em relação ao Gc. Com relação ao NGAL, houve aumento também do Gnt e do Gi em relação ao Gc. 0 Gt não diferiu dos demais. Conclui-se que a distensão do intestino delgado pode promover acúmulos de leucócitos nos cascos de equinos e que o NGAL é um método viável para se detectar infiltração neutrofílica em equinos. Novos estudos deverão ser realizados para se verificar possível benefício anti-inflamatório da hidrocortisona no casco de equinos com obstrução intestinal.

TERMOS DE INDEXAÇÃO: Equinos, laminite, calprotectina, NGAL, obstrução jejunal.

\section{INTRODUÇÃO}

A laminite é uma das principais doenças podais em equinos, que comumente leva a morte ou inabilidade esportiva, causando assim, importante perda econômica. Geralmente é observada como sequela de distúrbios gastrintestinais, respiratórios e reprodutivos que deflagram quadro inflamatório sistêmico (Eades et al. 2002, Parsons et al. 2007), afetando também animais com alterações endócrinas (Eades et al. 2002, Johnson et al. 2002), como portadores da síndrome de cushing, animais obesos ou que recebam altas dosagens de corticóides.

A fisiopatogenia da laminite parece estar relacionada à degradação da membrana basal (MB) por substâncias proteolíticas, e/ou perda dos hemidesmossomos, os quais fazem a união entre a lâmina densa da membrana basal e as células epidérmicas do tecido laminar (Pollitt \& Daradka 1998, French \& Pollitt 2004). A membrana basal se localiza entre as lâminas dérmicas e epidérmicas, sendo que estas estruturas formam interdigitações responsáveis pela fixação da falange distal à parede do casco. A falência desta estrutura pode resultar em rotação e/ou afundamento da falange distal (Pollitt 1996), por causar ruptura da união tecidual. A MB é composta por laminina (glicoproteína) e colágeno tipos IV e VII (Pollitt 1994), sendo que estas substâncias sofrem degradação enzimática fisiológica, realizada na maioria das vezes por metaloproteinases, produzidas pelas células basais para o controle do crescimento normal do casco (Pollitt 1996, Pollitt 2003).

Há alguns anos, a patogenia da laminite esteve associada à degradação do colágeno pela superativação das metaloproteinases presentes no tecido laminar (Pollitt 1994), constituindo a base da teoria enzimática. A ativação destas enzimas ainda é pouco conhecida, sugerindo-se o envolvimento de toxinas bacterianas, absorvidas do trato gastrintestinal, e de citocinas inflamatórias deflagradas nos quadros de ativação inflamatória sistêmica, como o fator de necrose tumoral alfa (TNF- $\alpha$ ) (Pollitt 1999, Han et al. 2001), e interleucinas (IL), como a IL-6 (Visser \& Pollitt 2011a). No entanto, alguns estudos têm demonstrado que a participação efetiva destas proteases no desenvolvimento da laminite pode ser questionável, principalmente da MMP-2, uma vez que nem sempre o aumento está associado com as alterações morfológicas observáveis (Visser \& Pollitt 2011b).

Os achados de vários estudos realizados no período de desenvolvimento da laminite induzida fornecem funda- mentos sobre o envolvimento da ativação inflamatória sistêmica, e não apenas local, na patogenia da laminite, como a presença de citocinas inflamatórias circulantes (Loftus et al. 2007, Noschka et al. 2009) e espécies reativas de oxigênio (Hurley et al. 2006) além de infiltração leucocitária tecidual, a qual pôde ser observada graças a implementação de técnicas laboratoriais específicas, principalmente a imunoistoquímica (Black et al. 2006, Hurley et al. 2006, Loftus 2006, Faleiros 2009). A explicação para a ativação inflamatória sistêmica pode ser de origem infecciosa, como por endotoxinas, as quais foram detectadas no início da doença por Bailey et al. (2007) dentre outros, ou injúria tecidual, observada no quadro de abdômen agudo (Eades 2002, Rio Tinto et al. 2004), ambas promovendo o quadro inflamatório.

As evidências de envolvimento inflamatório na fase de desenvolvimento da laminite permitem associar a doença com a falência de múltiplos órgãos no homem, onde a resposta inflamatória sistêmica leva à injúria de locais distantes ao sítio da afecção (Belknap, Moore \% Crouser 2009). Neste contexto, a infiltração leucocitária, observada nos períodos iniciais de desenvolvimento da laminite (Black et al. 2006, Hurley et al. 2006, Faleiros et al 2011a, Visser \& Pollitt 2011c) pode contribuir para a patogenia da laminite pela liberação de substâncias lesivas dos leucócitos aos tecidos, como as MMPs (Stetler-Stevenson 1990), mieloperoxidase e espécies reativas de oxigênio (Belknap \& Black 2005, Hurley et al. 2006, Riggs et al. 2007). Nesta linha de pensamento é de se esperar que nas obstruções intestinais, observadas em casos de cólica e nas quais a laminite é uma das principais complicações, haja ativação sistêmica de neutrófilos com subsequente migração para o tecido laminar.

Apesar de evidências de que animais acometidos com cólicas fatais desenvolvem lesões laminares mesmo sem sinais clínicos de laminite (Laskoski et al 2009) e de que a distensão intestinal resulta em acúmulo de neutrófilos nos pulmões (Faleiros et al. 2008), até o presente momento não foram encontrados, na literatura disponível, estudos demonstrando acúmulo de neutrófilos nos cascos decorrente de obstrução intestinal. A lipocalina associada à gelatinase de neutrófilos (NGAL), é uma proteína liberada pelos neutrófilos (Borregaard \& Cowland 1997), produzida nos precursores de polimorfonucleares na medula óssea e estocada até que a célula seja ativada (Flower 1996). Esta proteína tem sido encontrada em tecidos sob estresse em associação com a MMP-9 (Gupta et al. 2007, Lorencini et al. 2009) provavelmente com a função de proteger a MMP9 contra sua autodegradação, contribuindo para um período enzimático proteolítico ativo mais longo (Gupta et al. 2007). Muitos estudos sugerem que o NGAL possa ser marcador de lesões e da infiltração neutrofílica em diversos órgãos, (Bu et al. 2006, Hemdahl et al. 2006, Gupta et al. 2007, Smith et al. 2008), predizendo, inclusive, o agravamento do quadro clínico (Bolignano et al. 2009).

Pelo mesmo raciocínio, a administração de anti-inflamatórios poderia impedir o desenvolvimento da laminite, face a presença significativa de mediadores inflamatórios na fase prodrômica da doença, atenuando assim seus efei- 
tos adversos (Campebell et al. 2007). A utilização de corticóides tem sido inicialmente desestimulada, pois muitas vezes o desenvolvimento de laminite é observado em animais que necessitam de doses altas e/ou repetidas desses medicamentos (Pollitt 1999, Johnson 2003, Ryu et al. 2004). A administração diária de corticóides causa resistência à insulina, a qual também apresenta relação com 0 desenvolvimento de laminite (Bailey et al. 2007, Tiley et al. 2007). No entanto, diversos estudos tem demonstrado redução significativa de mediadores inflamatórios em pacientes sépticos (Brasil et al. 1999, Annane \& Cavaillo 2003, Kumar et al. 2009, Yazar et al. 2010), o que pode sugerir efeito benéfico também para a laminite, uma vez que ela ocorra secundária a doenças com envolvimento inflamatório sistêmico.

O objetivo deste trabalho foi verificar indicadores do acúmulo de neutrófilos no tecido laminar de equinos submetidos a distensão do intestino delgado, tratados ou não com hidrocortisona.

\section{MATERIAL E MÉTODOS}

\section{Animais}

Neste trabalho foram utilizados fragmentos de tecido laminar de vinte equinos hígidos, adultos, sem raça definida, machos ou fêmeas e sem alterações locomotoras. 0 grupo controle (Gc) foi constituído de sete animais oriundos de abatedouro comercial ${ }^{6}$ e os demais grupos constituídos de tecido laminar de animais utilizados por outro estudo ${ }^{7}$; estes grupos experimentais foram distribuídos ao acaso, sendo: grupo instrumentado (Gi), contendo cinco animais, cujo procedimento cirúrgico envolveu manipulação das alças para localizar o segmento onde foi feita a incisão do jejuno para colocação do balão de látex, sem distendê-lo; grupo tratado (Gt), constituído por quatro animais que foram submetidos à enterotomia para colocação e insuflação do balão de látex, produzindo obstrução jejunal, e administração única de hidrocortisona (Solu-Cortef, Rhodia Farma) na dose de $4 \mathrm{mg} / \mathrm{kg}$ pela via intravenosa; e por fim, o grupo não tratado (Gnt), constituído por quatro animais, submetidos à distensão jejunal pelo balão, sem receber nenhum tratamento.

\section{Delineamento experimental}

Antes do procedimento cirúrgico, os animais foram submetidos a jejum alimentar e hídrico. A sedação foi feita com cloridrato de xilazina a $10 \%$ (Sedazine, Fort Dodge) e butorfanol (Torbugesic, Fort Dodge), administrados por via intravenosa. A anestesia local foi realizada pela técnica de "L" invertido, com cloridrato de lidocaína $2 \%$ (Xilestesin, Cristália), sendo o terço médio do jejuno exposto por meio da fossa paracostal esquerda, em posição quadrupedal, e submetido à enterotomia seguida da compressão da parede intestinal, mediante a implantação de um balão de látex vazio no lúmen (Faleiros et al. 2002). 0 balão foi inflado com ar, até atingir a pressão de $12 \mathrm{mmHg}$, e retirado após quatro horas de obstrução intestinal, por laparotomia exploratória pelo flanco. Após 18 horas de reperfusão circulatória os animais foram submetidos à eutanásia por superdosagem anestésica (Thiopentax, Cristália).

\footnotetext{
${ }^{6}$ Abatedouro e Frigorífico Pomar, Araguari, Minas Gerais, Brasil.

${ }^{7}$ Material obtido de outro estudo, denominado "Obstrução experimental de jejuno de equinos tratados ou não com hidrocortisona: aspectos clínicos, hematológicos, bioquímicos e anatomopatológicos", aprovado pela Comissão de Ética e Bem Estar Animal, FCAV-Unesp, Jaboticabal, São Paulo (Protocolo no. 013598-06).
}

\section{Obtenção dos fragmentos laminares do casco}

Após a desarticulação metacarpo-falangeana de ambos os membros torácicos com um instrumento cortante, o casco foi seccionado no plano medial (Pollitt 1996), retirando-se fragmentos lamelares com o auxílio de uma pinça e lâmina de bisturi. Os fragmentos foram fixados em formol a $10 \%$ por um período de 24 horas, desidratadas em álcool e incluídos em parafina, para a análise imunoistoquímica. Ainda, foi realizado o congelamento de fragmentos do tecido laminar imediatamente após a coleta, pela imersão em nitrogênio líquido e posterior acondicionamento em freezer a menos $70^{\circ} \mathrm{C}$, para realização da zimografia.

\section{Imunoistoquímica para pesquisa de calprotectina}

Foram feitos cortes teciduais a $5 \mu \mathrm{m}$ de espessura, desparafinados em estufa a $60^{\circ} \mathrm{C}$ (1 hora) e hidratados com banhos em xilol e álcool, em concentrações decrescentes. Para recuperação antigênica utilizou-se Proteinase $\mathrm{K}(20 \mu / \mathrm{mL})$ e o bloqueio da peroxidase endógena foi realizado com peroxido de hidrogênio a $3 \%$, diluído em metanol. Os cortes foram incubados em estufa a $37^{\circ} \mathrm{C}$ com anticorpo primário anti-calprotectina ${ }^{8}$, produzido em camundongo por 1 hora e 30 minutos, na diluição 1:250. Foi utilizado controle secundário biotinilado na diluição 1:100, com incubação em temperatura ambiente por 30 minutos. 0 método utilizado foi o complexo ABC (Vectastain ${ }^{\circledR}$ Elite ABC Kit, Vector, USA), sendo os cortes incubados em temperatura ambiente por mais 30 minutos. 0 cromógeno utilizado foi substrato de peroxidase $^{9}$ por quatro minutos. Na contra-coloração utilizou-se a Hematoxilina de Harris.

A pesquisa de leucócitos mielóides foi realizada pela contagem de células positivas para o anticorpo, em microscópio óptico com aumento de $10 \mathrm{x}$, contando 20 campos por lâmina. Nas lâminas cuja área de corte era pequena, foram contados todos os campos. Foi utilizado o microscópio CH30 RF100-Japan, e a objetiva foi NCWHK 10x (FN 18), cujo campo de visão no aumento de 10x possui $1,8 \mathrm{~mm}$ de diâmetro, ou seja, uma área de $2,54 \mathrm{~mm}^{2}$.

\section{Zimografia}

Os fragmentos do tecido laminar congelados foram triturados para a extração das proteínas em tampão contendo $50 \mathrm{mM}$ Tris- $\mathrm{HCl} \mathrm{pH} \mathrm{7,4;} \mathrm{0,2M} \mathrm{NaCl;} \mathrm{0,1 \%} \mathrm{Triton;} 10 \mathrm{mM} \mathrm{CaCl} 2$ e 1\% protease inhibitor cocktail ${ }^{10}$. A quantificação protéica foi realizada de acordo com Bradford (1976), utilizando uma curva padrão de soro albumina bovino (BSA).

A zimografia para pesquisa do NGAL foi realizada em gel de poliacrilamida $10 \%$ contendo gelatina $0,1 \%$. Aproximadamente $20 \mu \mathrm{g}$ de proteína por amostra foram aplicadas no gel. Após eletroforese, o gel foi lavado à temperatura ambiente com 2,5\% Triton X-100, incubado overnight (15 horas aproximadamente) em tampão $50 \mathrm{mM}$ Tris-HCl, pH 7.4 contendo $0,1 \mathrm{M} \mathrm{NaCl} \mathrm{e} 0,03 \%$ azida sódica, a $37^{\circ} \mathrm{C}$. 0 gel foi corado com o corante Coomassie brilliant blue._A banda correspondente à atividade gelatinolítica do NGAL pode ser observada após lavagem com solução contendo metanol (30\%) e ácido acético (10\%). Os géis foram avaliados pela densitometria das bandas por meio do programa Scion Image.

\section{Estatística}

Para cada indivíduo, considerou-se a média dos resultados obtidos nos membros torácicos direito e esquerdo. A comparação entre grupos foi realizada por análise de variância em delinea-

\footnotetext{
${ }^{8}$ MAC387, Abcam, Cambridge, MA.

${ }^{9}$ Vector ${ }^{\circledR}$ NovaRed ${ }^{\mathrm{TM}}$, SK-4800, red, Vector, EUA.

${ }^{10}$ Sigma Chemical Company, Saint Louis, MO, EUA.
} 
mento inteiramente casualizado, sendo as médias comparadas pelo teste de Duncan (imunoistoquímica) ou de Tukey (zimografia), considerando-se $\mathrm{P} \leq 0,05$.

\section{RESULTADOS}

A imunomarcação para calprotectina detectou células positivas na derme em animais de todos os grupos experimentais. Esta marcação foi dada como positiva ao visualizar células com coloração marrom. Não foi verificado em nenhum dos animais imunomarcação difusa em células epidermais, como demonstrado na Figura 1 . As médias $( \pm$ EPM) foram 0,29 $\pm 0,06 \mathrm{em} \mathrm{Gc}, 0,33 \pm 0,08 \mathrm{em} \mathrm{Gi}, 0,60 \pm 0,12$

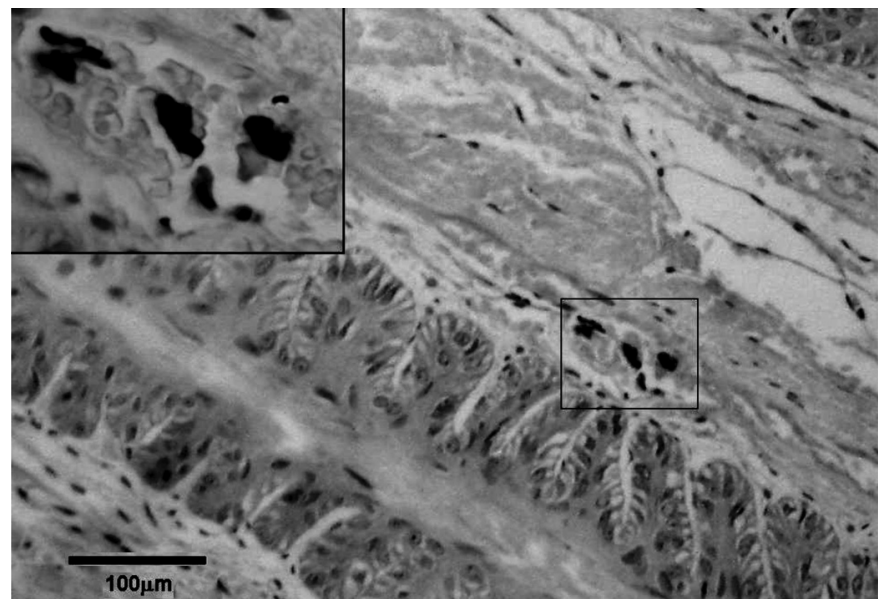

Fig.1. Tecido laminar do casco de um equino do grupo não tratado, mostrando leucócitos positivos para calprotectina (detalhe) na lâmina dérmica primária. Imunoistoquímica, método $A B C$, cromógeno substrato de peroxidase, obj.20x.

em Gnt e 0,70 $\pm 0,19$ em Gt. As contagens de células positivas foram maiores nos dois grupos distendidos, tratado e não tratado, em relação ao Gc, contudo sem diferença entre eles (Fig.2). A lipocalina associada à gelatinase de neutrófilos (NGAL), demonstrada pela conjugação desta substância à MMP-9 (metaloproteinase-9) foi observada nos géis de poliacrilamida com densitometria de $135 \mathrm{kDa}$ (Fig.3). A presença da substância foi vista em todos os grupos submetidos ao procedimento cirúrgico apenas, não se observando a presença da mesma no grupo controle. $\mathrm{Na}$ avaliação estatística, não houve diferenças entre os grupos submetidos ao procedimento cirúrgico, grupo instrumentado (Gi), grupo não tratado (Gnt) e grupo tratado (Gt). Os grupo Gi e Gnt apresentaram aumento do NGAL em relação ao grupo controle (Gc); 0 grupo tratado (Gt) não apresentou diferenças com os demais, tampouco com o grupo controle (Gc) (Fig.4).

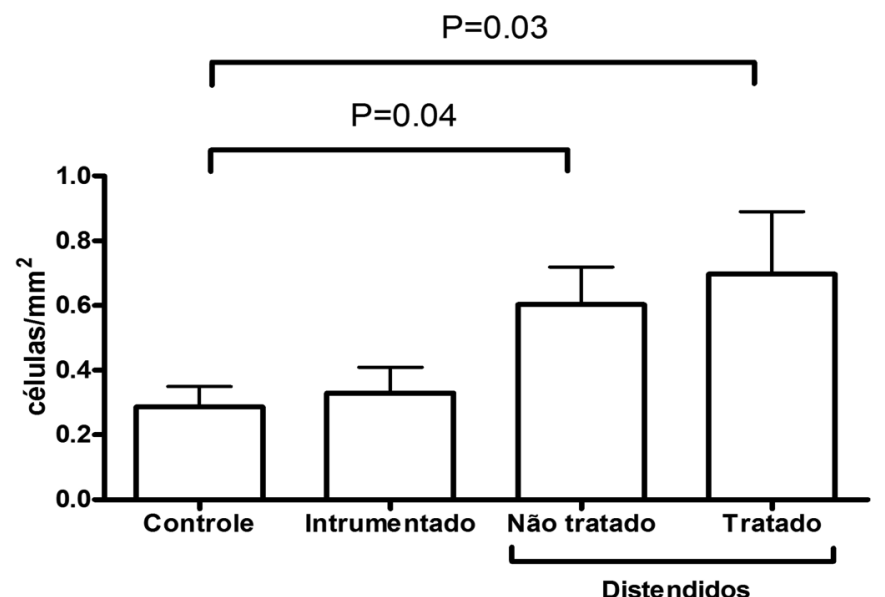

Fig.2. Médias ( \pm EPM) das contagens de leucócitos positivos para calprotectina em equinos submetidos a distensão do jejuno, tratados ou não com hidrocortisona.

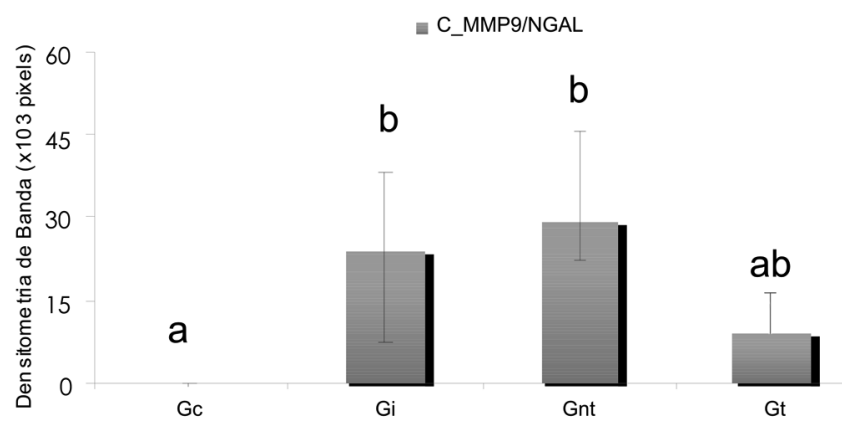

Fig.4. Médias e desvios-padrão da densitometria de bandas para o complexo MMP-9/NGAL (C_MMP-9/NGAL) entre os grupos controle e experimentais, e respectivo desvio padrão. Colunas marcadas pela mesma letra não diferem entre si.

\section{DISCUSSÃO}

0 modelo de injuria intestinal vem sendo utilizado, em outras espécies, como indutor de sepse, a qual está intrinsecamente relacionada à inflamação sistêmica (Benjamin 2001), e pode, ainda, ocorrer pelas lesões de isquemia-reperfusão intestinal (Bianco-Blatlles et al. 2008), as quais provavelmente ocorreram durante a obstrução luminal dos animais deste estudo. Semelhante a diversos trabalhos realizados com indução experimental de laminite, neste trabalho também houve aumento da quantidade de leucócitos no tecido laminar do casco, tanto por meio da zimografia como de imunoistoquímica. Faleiros et al. (2008) relataram, em quadro semelhante, que a distensão do cólon menor induziu acúmulo pulmonar de neutrófilos, suportando a hipótese de indução de lesão remota em seguida à resposta inflamatória sistêmica, com ou sem sepse. Apesar de

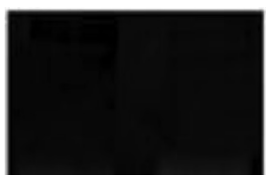

Gc

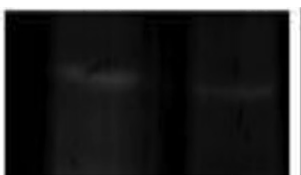

$\mathrm{Gi}$

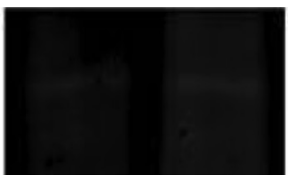

Gnt

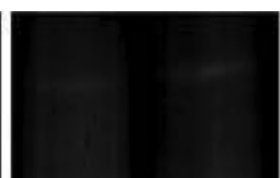

Gnt

Fig.3. Atividade enzimática das MMPs, registrada em gel de poliacrilamida. Nota-se que somente as amostras dos grupos Gi, Gnt e Gt possuem a banda formada pela substância MMP-9 conjugada a NGAL (C_MM)-9/NAGL). 
não ser um modelo clássico de indução da afecção podal, é interessante por tratar-se de lesões causadas no aparelho gastrintestinal que repercutem a distância nas lâminas do casco, semelhante ao observado por Rio Tinto et al. (2004).

A MMP-9 tem sido associada ao desenvolvimento da injúria tecidual em vários órgãos, principalmente pela sua forte associação com a infiltração neutrofílica (Loftus et al. 2006, Chakrabarti, Zee \& Patel 2006, Mikami et al. 2009). Neste estudo, houve aumento da forma de MMP-9 conjugada ao NGAL (C_MMP-9/NGAL), um composto associado a neutrófilos, o que suporta a participação da MMP-9 na resposta inflamatória. De Laat et al. (2011) demonstraram por PCR e zimografia que dentre várias metaloproteinases e seus inibidores, a única substância que apresentou aumento significativo em cavalos após 48 horas de indução de laminite foi a MMP-9.

A técnica de imunoistoquímica empregada confirmou a presença de células inflamatórias no tecido laminar do casco (Black et al. 2006, Faleiros et al. 2009), sendo que a MMP-9 conjugada ao NGAL, observada na zimografia, foi relacionada, por outros estudos, à infiltração neutrofílica tecidual, utilizada como biomarcador de lesão tecidual em vários órgãos, e de neutrófilos (Nielsen et al. 1996, Hemdahl et al. 2006, Gupta et al. 2007, Bu et al. 2008, Smith et al. 2008, Zabron et al. 2011). Shapiro et al. (2009) observaram que sua apresentação como biomarcador mostrou-se confiável para sepse, choque séptico e morte nos pacientes com comprometimento sistêmico. A ausência desta substância no grupo controle também pode estar associada à baixa marcação imunoistoquímica do mesmo, o qual supostamente não possui leucócitos ativados no tecido laminar (Loftus et al. 2006). É importante salientar que o NGAL apresenta marcação para neutrófilos, e talvez por isso não tenha sido observado no grupo controle, uma vez que a calprotectina marca não apenas neutrófilos, mas também monócitos, os quais já foram demonstrados por Faleiros et al. (2011b) como presentes em cavalos normais.

As contagens de leucócitos positivos para calprotectina no grupo controle $\left(0,29 \pm 0,11\right.$ células $\left./ \mathrm{mm}^{2}\right)$ neste trabalho foram muito próximas das descritas por Faleiros et al. (2011a) em animais normais $\left(0,4 \pm 0,3\right.$ células $\left./ \mathrm{mm}^{2}\right)$. Tais autores demonstraram aumento nas contagens destas células no tecido laminar, chegando em média a 3,1 células $/ \mathrm{mm}^{2}$ na fase de desenvolvimento e a 43,4 células $/ \mathrm{mm}^{2}$ no início dos sinais clínicos , indicando seu possível papel no desenvolvimento da doença. No presente estudo houve aumento nas contagens de leucócitos nos animais dos grupos distendidos, contudo as médias $\left(0,6-0,7\right.$ células $\left./ \mathrm{mm}^{2}\right)$ estiveram abaixo dos valores relatados em animais com laminite induzida por excesso de carboidrato (Faleiros et al. 2011a).

Estes achados, associados à ausência de imunomarcação positiva para calprotectina na epiderme e de lesões histológicas evidentes, sugerem que a resposta inflamatória promovida pela distensão do jejuno foi suficiente para promover acúmulo leucocitário remoto nos cascos, mas que às 18 horas de reperfusão, quando as amostras foram colhidas, o insulto ainda era insuficiente para induzir sinais evidentes do processo de desenvolvimento de laminite.

Black et al. (2006), analisando a microvasculatura dérmica do tecido laminar do casco de animais com laminite induzida por extrato de nogueira preta, observaram aumento no número de leucócitos, indicando similaridade com a falência orgânica que ocorre na sepse humana, onde a migração leucocitária desempenha papel lesivo significativo. Porém existem evidências de que o processo inflamatório que precede a doença é sistêmico, e não apenas local, com ativação de leucócitos sanguíneos (Hurley et al. 2006). Leise et al. (2011) observaram que ocorre aumento da expressão genética de diversos fatores inflamatórios, como as interleucinas IL-1 $\beta$, IL-6 e a ciclooxigenase 2 , em equinos após a indução de laminite por administração de carboidrato. Contribuindo também para esta hipótese, o estudo de Visser \& Pollitt (2011c) demonstrou que a degradação do colágeno tipo IV, presente no tecido laminar do casco, ocorre em outros locais do organismo de um cavalo com laminite, como pele e estômago, o que sugere fator desencadeante sistêmico para a doença.

A administração de glicocorticóides, em altas doses e por períodos prolongados pode levar ao desenvolvimento da laminite (Johnson et al. 2002) provavelmente devido ao aumento da insulina (Tylei et al. 2007). Suagee et al. (2011) observaram, após a indução da laminite em cavalos, que houve aumento da insulina, e que este aumento esteve associado com o desenvolvimento de resposta inflamatório sistêmica. Ainda, outros estudos relacionam a elevação da insulina com disfunção circulatória (Venugopal et al. 2011), o que pode causar redução da perfusão sanguínea do casco (Cornelisse et al. 2006). Ryu et al (2004) relataram o desenvolvimento de laminite aguda e hepatopatia em um equino pela administração de triamcilonona durante dez dias consecutivos. No entanto, os achados do estudo atual tendem a mostrar efeito positivo do tratamento preventivo com hidrocortisona em equinos com obstrução intestinal, em dose única de $4 \mathrm{mg} / \mathrm{kg}$, pois não houve aumento no NGAL em relação ao controle no grupo tratado.

Aljada et al. (2001) observaram que a administração de hidrocortisona inibe a atividade das MMPs 2 e 9, ambas enzimas proteolíticas. Achados de Brasil et al. (1999) e Annane \& Cavaillo (2003) demonstraram que a utilização de corticosteróides inibe a liberação de citocinas inflamatórias como o TNF- $\alpha$, reduzindo a proliferação e infiltração leucocitária, o que tornaria seu uso benéfico em modelos de sepse. Sabe-se que a infiltração leucocitária está associada a lesões em diversos tecidos, mas especialmente o renal e pulmonar (Bai et al. 2009, Kumar et al. 2009, Maisel et al. 2011). Existem diversos trabalhos que obtiveram redução de mediadores inflamatórios com a utilização de corticóides. Kumar et al. (2009) observaram que a dexametasona administrada em ratos antes e após isquemia e reperfusão renal foi capaz de proteger o órgão contra a injúria, reduzindo inclusive a creatinina sérica, quando comparado ao grupo controle, bem como redução marcante na infiltração leucocitaria renal. Green et al. (2009), trabalhando com pessoas portadoras de meningite tuberculosa, constataram que o efeito benéfico que a dexametasona exerce sobre a evolução da doença podem ser devidos a redução da MMP-9 e da contagem de neutrófilos no fluido cérebro-espinhal dos pacientes, os quais encontravam-se fortemente correlacionados. Sun et al. (2007), trabalhando com indução de pancreatite em ratos e 
administração de hidrocortisona, obtiveram injúria pulmonar superior no grupo controle, que não recebeu a droga, antes da indução da lesão, o que sugere efeito protetor preventivo. Estudos realizados por Yazar et al.(2010) demonstraram que a administração de dexametasona em alta dose, associada ao flunixim meglumine, em ratos com endotoxemia induzida, apresentam maior efetividade em reduzir citocinas inflamatórias do que baixa dose de dexametasona ou os tratamentos individualizados.

Estes resultados fornecem apenas indícios de que a hidrocortisona possa trazer benefício para a prevenção da laminite. Embora os glicocorticóides sejam vistos com ressalvas no tratamento de pacientes críticos, tanto na medicina humana quanto na medicina veterinária, deve-se destacar que a administração deste medicamento em uma única aplicação e baixa dosagem parece ter reduzido o aumento de NGAL nos grupos submetidos à distensão de jejuno. Isto indica que este fármaco possa ser administrado em equinos com afecções agravadas pela ativação sistêmica, com o intuito de coibir seus efeitos deletérios como redução de mediadores inflamatórios e do estresse oxidativo (Kaufmann et al. 2008). Uma vez comprovada a efetiva participação dos leucócitos na fisiopatogenia da laminite, este estudo pode indicar possível papel protetor da integridade laminar do casco. Para tanto, mais estudos devem ser realizados.

\section{CONCLUSÕES}

A distensão do jejuno promove acúmulo de leucócitos no tecido laminar do casco, indicando que estas células estão envolvidas no desenvolvimento da laminite como complicação de distúrbios gastrintestinais.

O NGAL pode ser utilizado para identificar a presença de neutrófilos no tecido laminar do casco de equinos, auxiliando nos estudos sobre a enfermidade podal.

A hidrocortisona, na dose de $4 \mathrm{mg} / \mathrm{kg}$, e em dose única, pode auxiliar na prevenção das lesões responsáveis pela laminite durante a fase prodrômica da doença, reduzindo a expressão de NGAL no tecido laminar do casco.

\section{REFERÊNCIAS}

Aljada A., Ganhim H., Mohanty P., Hofmeyer D., Tripathy D. \& Dandona P. 2001. Hydrocortisone suppresses intranuclear activator-protein-1 (AP-1) binding activity in mononuclear cells and plasma matrix metalloproteinase 2 and 9 (MMP-2 and MMP-9). J. Clin. Endocrinol. Metabol. 86(12):5988-5991.

Annane D. \& Cavaillon J.M. 2003. Corticosteroids in sepsis: From bench to bedside? Shock 20:197-207.

Bai J.W., Deng W.W., Lin M.J., Xu S.M. \& Zhang D.X. 2009. The effects of vitamin $E$ and dexamethasone on inflammation of acute lung injury and expression of myosin light chain kinase. Chinese J. Tubercul. Resp. Dis. 32(1):46-50.

Bailey S.R., Menzies-Gow N.J., Harris P.A., Habershon-Butcher J.L., Crawford C., Berhane Y., Boston R.C. \& Elliott J. 2007. Effect of dietary fructans and dexamethasone administration on the insulin response of ponies predisposed to laminitis. J. Am. Vet. Med. Assoc.. 231(9):1365-1373.

Belknap J., Moore J.N. \& Crouser E.C. 2009. Sepsis: From human organ failure to laminar failure. Vet. Immun. Immunopathol. 129(3/4):155-157.

Belknap J.K. \& Black S.J. 2005. Review of the pathophysiology of the developmental stages of equine laminitis. American Association of Equine Pratitioners. Disponível em <http://www.ivis.org/proceedings/ aaep/2005/belknap/chapter.asp?LA=1> Accessed on Oct. 31, 2008.

Benkamin C.F. 2001. Atualização sobre mediadores e modelos experimentais de sepse. Medicina, Ribeirão Preto, 34:18-26.
Bianco-Blatlles M.D., Sosunov A., Polin R.A. \& Ten V.S. 2008. Systemic inflammation following hind-limb ischemia- reperfusion affects brain in neonatal mice. Developmental Neuroscience 30(6):367-373.

Black S.J., Lunn D.P., Yin C., Hwang M., Lenz S.D. \& Belknap J.K. 2006. Leukocyte emigration in the early stages of laminitis. Vet. Immun. Immunopathol. 109:161-166.

Bolignano D., Basile G., Parisi P., Coppolino G., Nicocia G. \& Buemi M. 2009. Increased plasma neutrophil gelatinase-associated lipocalin levels predict mortality in elderly patients with chronic heart failure. Rejuvenation Res. 12(1):1-7

Borregaard N. \& Cowland J.B. 1997. Granules of the human neutrophilic polymorphonuclear leukocyte. Blood 89:3503-3521.

Brasil L.A., Gomes W.J., Salomão R., Fonseca J.H.P., Branco J.N.R. \& Buffolo E. 1999. Uso de corticóide como inibidor da resposta inflamatória sistêmica induzida pela circulação extracorpórea. Revta Bras. Cirur. Cardiovasc. 14(3):254-268.

Bradford M.M. 1976. A rapid and sensitive method for quantitation of microgram quantities of protein utilizing the principle of protein-dye-binding. Analytical. Biochem. 72:248-254.

Bu D., Hemdahl A.L., Gabrielsen A., Fuxe J., Zhu C., Eriksson P. \& Yan Z.Q. 2006. Induction of neutrophil gelatinase-associated lipocalin in vascular injury via activation of nuclear factor-kB. Am. J. Pathol. 169:2245-2253.

Campebell R.C., Peiro J.R., Rosa P.C.S., Valadao C.A.A. \& Bechara G.H. 2007. Endotoxemia por lipopolissacarídeo de Escherichia coli, em equinos: efeitos de antiinflamatórios nas concentrações sérica e peritonial do fator de necrose tumoral alfa (TNF- $\alpha$ ). Arq. Bras. Med. Vet. Zootec. 59(4):837-843.

Chakrabarti S., Zee J.M. \& Patel K.D. 2006. Regulation of matrix metalloproteinase-9 (MMP-9) in TNF-stimulated neutrophils: Novel pathways for tertiary granule release. J. Leukocyte Biology 79:214-222.

Cornelisse C.J., Robinson N.E., Berney C.A., Eberhart S., Hauptman J.E. \& Derksen F.J. 2006.Thermographic study of in vivo modulation of vascular responses to phenylephrine and endothelin-1 by dexamethasone in the horse. Equine Vet. J. 38(2):119-126.

De Laat M.A., Kyaw-Tanner M.T., Nourian A.R., McGowan C.M., Sillence M.N. $\&$ Pollitt C.C. 2011. The developmental and acute phases of insulin-induced laminitis involve minimal metalloproteinase activiy. Vet. Immunol.. Immunopathol. 140(3/4):275-281.

Eades S.C., Holm A.M.S. \& Moore R.M. 2002. A Review of the pathophysiology and treatment of acute laminitis: phatophysiologic and therapeutic implications of endothelin-1. Am. Assoc. Equine Pract. 48:353-361.

Faleiros R.R., Macoris D.G., Alessi A.C., Saquetti C.H. \& Rasera L. 2002. Effect of intraluminal distention on microvascular perfusion in the equine small colon. Am. J. Vet. Res. 63:1292-1297.

Faleiros R.R., Macoris D.G., Alves G.E., Souza D.G., Teixeira M.M. \& Moore R.M. 2008. Local and remote lesions in horses subjected to small colon distension and decompression. Can. J. Vet. Res. 72(1):68-76.

Faleiros R.R., Nouvo G.J. \& Belknap J.K. 2009. Calprotectin in myeloid and epithelial cells of laminae from horses with black walnut extractinduced laminitis. J. Vet. Intern. Med. 23(1):174-181.

Faleiros R.R., Johnson P.J., Nuovo G.J., Messer N.T., Black S.J. \& Belknap J.K. 2011a. Laminar leukocyte accumulation in horses with carbohydrate overload induced laminitis. J. Vet. Intern. Med. 25(1):107-15.

Faleiros R.R., Nuovo G.J., Flechtner A.D. \& Beljnap J.K. 2011b. Presence of mononuclear cells in normal and affected laminae from the black walnut extract model of laminitis. Equine Vet. J. 43(1):45-53.Flower D.R. 1996. The lipocalin protein family: structure and function. Biochem. J. 318:1-14.

French R.K. \& Pollitt C. 2004. Equine laminitis: loss of hemidesmosomes in hoof secondary epidermal lamellae correlates to dose in an oligofructose induction model: a ultrastructural study. Equine Vet. J. 36(3):230-236.

Green J.A., Tran C.T.H., Farrar J.J., Nguyen M.T.H., Nguyen P.H., Dinh S.X., Ho N.D.T., Ly C.V., Tran H.T., Friedlan J.S. \& Thwaites G.E. 2009. Dexamethasone, cerebrospinal fluid matrix metalloproteinase concentrations and clinical outcomes in tuberculous meningitis. PloS ONE 4(1):7277.

Gupta K., Shukla M., Cowland J.B., Malemud C.J. \& Haqq T.M 2007. Neutrophil gelatinase-associated lipocalin is expressed in osteoarthritis and 
forms a complex with matrix metalloproteinase 9. Arthritis and Rheumatism 56(10):3326-3335.

Han Y., Tuan T.L., Wu H., Hughes M. \& Garner W.L. 2001. TNF- $\alpha$ stimulates activation of pro-MMP-2 in human skin through NF- $\kappa B$ mediated induction of MT1-MMP. J. Cell Sci. 114:131-139.

Hemdahl A.L., Gabrielsen A., Zhu C., Eriksson P., Hedin U., Kastrup J., Thorén P. \& Hansson G.K. 2006. Expression of neutrophil gelatinase-associated lipocalin in atherosclerosis and myocardial infarction. Arteriosclerosis Thrombosis and Vascular Biology 26(1):136-142.

Hurley D.J., Parks R.J., Reber A.J., Donovan D.C., Okinaga T., Vandenplas M.L., Peroni J.F. \& Moore J.N. 2006. Dynamic changes in circulating leukocytes during the induction of equine laminitis with black walnut extract. Vet. Immunol. Immunopathol. 110(3/4):195-206.

Johnson P.J., Slight S.H., Ganjam V.K. \& Kreeger J.M. 2002. Glucocorticoids and laminitis in the horse. Vet. Clin. North Am., Equine Pract. 18(2):219-236.

Johnson P.J., Bowles D.K. \& Messer N.T. 2003. Dexamethasone alters equine digital artery smooth muscle ion channel activity and contractility: Role in glucocorticoid associated laminitis? J. Vet. Intern. Med. 17(3):457-458.

Kaufmann I., Briegel J., Schliephake F., Hoelzl A., Chouker A., Hummel T., Schelling G. \& Thiel M. 2008. Stress doses of hydrocortisone in septic shock: Beneficial effects on opsonization-dependent neutrophil functions. Intensive Care Medicine 34(2):344-349.

Kumar S., Allen D.A., Kieswich J.E., Patel N.S.A., Harwood S., Mazzon E., Cuzzocrea S., Raftery M.J., Thiemermann C. \& Yaqoob M.A. 2009. Dexamethasona ameliorates renal ischemia-reperfusion injury. J. Am. Soc. Nephronol. 20(11):2412-2425.

Laskoski L.M.,Valadão C.A.A., Vasconcelos R.O., Pereira R.N., Dias A.D.P.U. \& Alessi A.C . 2009. Alterações morfológicas de tecido laminar do casco e parâmetros clínicos e laboratoriais de equinos com síndrome cólica letal. Pesq. Vet. Bras. 29(9):731-735.

Leise B.S., Faleiros R.R., Watts M., Johnson P.J., Black S.J. \& Belknap J.K. 2011. Laminar Inflammatory gene expression in the carbohydrate overload model of equine laminitis. Equine Vet. J. 43(1):54-61.

Loftus J.P., Belknap J.K. \& Black S.J. 2006. Matrix metalloproteinase-9 in laminae of black walnut extract treated horses correlates with neutrophil abundance. Vet. Immunol. Immunopathol. 113:267-276.

Loftus J.P., Black S.J., Pettigrew A., Abrahansen E.J. \& Belknap J.K. 2007. Early laminar events involving endothelial activation in horses with black walnut-induced laminitis. Am. J. Vet. Res. 68(11):1205-1211.

Lorencini M., Silva J.A., De La Hoz C.L., Carvalho H.F. \& Stach-Machado D.R. 2009. Changes in MMPs in inflammatory cells in experimental gingivitis. Histology Histopathol. 24(2):157-166.

Maisel A.S., Mueller C., Fitzgerald R., Brikhan R., Hiestand B.C., Iqbal N., Clopton P. \& Van Veldhuisen D.J. 2011. Prognostic utility of plasma neutrophil gelatinase-associated lipocalin in patients with acute heart failure: the NGAL evaluation along with B-type natriuretic peptide in acutely decompensated heart failure (GALLANT) trial. Eur. J. Heart Failure 13(8):846-851.

Mikami Y., Dobschutz E.V., Sommer O., Wellner U., Unno M., Hopt U. \& Keck T. 2009. Matrix metalloproteinase-9 derived from polymorphonuclear neutrophils increases gut barrier dysfunction and bacterial translocation in rat severe acute pancreatitis. Surgery 145(2):147-156.

Nielsen B.S., Borregaard N., Bundgaard J.R., Timshel S., Sehested M. \& Kjeldsen L. 1996. Induction of NGAL synthesis in epithelial cells of human colorectal neoplasia and inflammatory bowel diseases. Gut 38:414-420.

Noschka E., Vandenplas M.L., Hurley D.J. \& Moore J.N. 2009. Temporal aspects of laminar gene expression during the developmental stages of equine laminitis. Vet. Immunol. Immunopathol. 129(3/4):242-253.

Parsons C.S., Orsini J.A., Krafty R., Capewell L. \& Boston R. 2007. Risk factors for development of acute laminitis in horses during hospitalization: 73 cases (1997-2004). J. Am. Vet. Med. Assoc. 230(6):885-889.

Pollitt C.C. 1994. The basement membrane at the equine hoof dermal epidermal junction. Equine Vet. J. 26(5):399-407.
Pollitt C.C. 1996. Basement membrane pathology: a feature of acute equine laminitis. Equine Vet. J. 28(1):38-46.

Pollitt C.C. \& Daradka M. 1998. Equine laminitis basement membrane pathology: loss of type IV collagen, type VII collagen and laminin immunostaining. Equine Vet. J. 26(Suppl.):139-144.

Pollitt C.C. 1999. Equine laminitis: A revised pathophysiology. Am. Assoc. Equine Pract. Proc. 45:188-192.

Pollitt C.C. 2003. Pathophysiology of laminitis, p.325-329. In: Dyson S.J. \& Ross M.W. (Eds), Diagnosis and Management of Lameness in the Horse. W.B. Saunders, Philadelphia.

Riggs L.M., Franck T., Moore J.N., Krunkosky T.M., Hurley D.J., Peroni J.F., De La Rebiere G. \& Serteyn D.A. 2007. Neutrophil myeloperoxidase measurements in plasma, laminar tissue, and skin of horses given black walnut extract. Am. J. Vet. Res. 68:81-86.

Rio Tinto J.J.M., Alves G.E.S., Faleiros R.R., Santos R.L.. Marques Júnior A.P. \& Melo E.G. 2004. Utilização de hidrocortisona em equinos submetidos a isquemia e reperfusão no jejuno e suas conseqüências sob o cório laminar. Arq. Bras. Med. Vet. Zootec. 56(3):292-299.

Ryu S., Kim B., Lee C., Yoon J. \& Lee Y. 2004. Glucocorticoide-induced laminitis with hepatopathy in a thoroughbred filly. J. Vet. Sci. 5(3):271274.

Shapiro N.I., Trzeciak S., Hollander J.E., Birkhahn R., Otero R., Osborn T.M., Moretti E., Nguyen H.B., Gunnerson K.J., Milzman D., Gaieski D.F., Goyal M., Cairns C.B., Ngo L. \& Rivers E.P. 2009. A prospective, multicenter derivation of a biomarker panel to assess risk of organ dysfunction, shock, and death in emergency department patients with suspected sepsis. Critical Care Medicine 37(1):96-104.

Smith E.R., Zurakowski D., Saad A., Scott R.M. \& Moses M.A. 2008. Urinary biomarkers predict brain tumor presence and response to therapy. Clin. Cancer Res. 14(8):2378-2386.

Stetler-Stevenson W.G. 1990. Type IV collagenases in tumor invasion and metastasis. Cancer and Metastasis Reviews 9(4):289-303.

Suagee J.K., Corl B.A., Crisman M.V., Hulver M.W., McCutcheon L.J. \& Geor R.J. 2011. Effects of acute hyperinsulinemia on inflammatory proteins in Horses. Vet. Immunol. Immunopathol. 142(3/4):141-146.

Sun W., Watanabe Y., Toki A. \& Wang Z.Q. 2007. Beneficial Effects of hydrocortisone in induced acute pancreatitis of rats. Chinese Med. J. 120(20):1757-1761.

Tiley H.A., Geor R.J. \& McCutcheon L.J. 2007. Effects of dexamethasone on glucose dynamics and insulin sensitivity in healthy horses. Am. J. Vet. Res. 68(7):753-759.

Venugopal C.S., Eades S., Holmes E.P. \& Beadle R.E. 2011. Insulin resistance in equine digital vessel rings: An in vitro model to study vascular dysfunction in equine laminitis. Equine Vet. J. 43(6):744-749.

Visser M.B. \& Pollitt C.C. 2011a. Lamellar leukocyte infiltration and involvement of IL-6 during oligofrutose-induced equine laminitis development. Vet. Immunol. Immunopathol. doi:10.1016/j.vetimm.2011. 07.016

Visser M.B. \& Pollitt C.C. 2011b. The timeline of metalloprotease eventis during Olifructose induced equine laminitis development. Equine Vet. J. doi:10.1111/j.2042-3306.2011.00393.x

Visser M.B. \& Pollitt C.C. 2011c. Immunohistochemical distribution of laminin-332 and collagen Type IV in the basement membrane of normal horses and horses with induced laminitis. J. Comp.Pathol. 145(1):80-87.

Yazar E., Bulbul A., Avci G.E., Er A., Uney K., Elmas M. \&Tras B. 2010. Effects of enrofloxacin, flunixim meglumine and dexamethasone on disseminated intravascular coagulation, cytokine levels and adenosine deaminase activity in endotoxaemia in rats. Acta Vet. Hungarica 58(3):357-367.

Zabron A.A., Horneffer-Van der Sluis V.M., Wadsworth C.A., Laird F., Gierula M., Thillainayagam A.V., Vlavianos P., Westaby D., Taylor-Robinson S.D., Edwards R.J. \& Khan S.A. 2011. Elevated levels of neutrophil gelatinase-associated lipocalin in bile from patients with malignant pancreatobiliary disease. Am. J. Gastroenterol. 106:1711-1717. 